Portland State University

PDXScholar

$4-17-2021$

\title{
Psychometric Properties of the Brief Version of the Questionnaire of Olfactory Disorders in Patients with Chronic Rhinosinusitis.
}

\author{
Jose L. Mattos \\ University of Virginia \\ Todd Bodner \\ Portland State University, tbodner@pdx.edu \\ Jess C. Mace \\ Oregon Health and Science University \\ Rodney J. Schlosser \\ Medical University of South Carolina \\ Daniel M. Beswick \\ University of Colorado
}

Follow this and additional works at: https://pdxscholar.library.pdx.edu/psy_fac

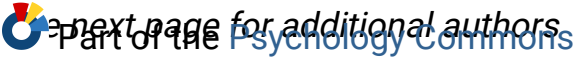

Let us know how access to this document benefits you.

\section{Citation Details}

Mattos, J. L., Bodner, T. E., Mace, J. C., Schlosser, R. J., Beswick, D. M., Ramakrishnan, V. R., Alt, J. A., Payne, S. C., Smith, T. L., \& Soler, Z. M. (2021). Psychometric properties of the brief version of the questionnaire of olfactory disorders in patients with chronic rhinosinusitis. International Forum of Allergy \& Rhinology, alr.22800. https://doi.org/10.1002/alr.22800

This Article is brought to you for free and open access. It has been accepted for inclusion in Psychology Faculty Publications and Presentations by an authorized administrator of PDXScholar. Please contact us if we can make this document more accessible: pdxscholar@pdx.edu. 


\section{Authors}

Jose L. Mattos, Todd Bodner, Jess C. Mace, Rodney J. Schlosser, Daniel M. Beswick, Vijay R.

Ramakrishnan, Jeremiah A. Alt, Spencer C. Payne, Timothy L. Smith, and Zachary M. Soler 


\title{
Psychometric properties of the brief version of the questionnaire of olfactory disorders in patients with chronic rhinosinusitis
}

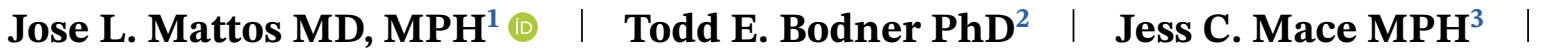 \\ Rodney J. Schlosser MD ${ }^{4,5}$ \\ Daniel M. Beswick MD ${ }^{6}$ (1)

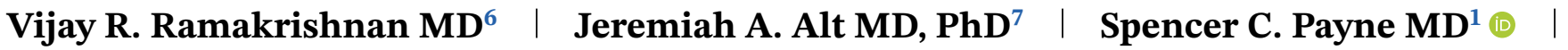 \\ Timothy L. Smith MD, MPH ${ }^{3}$ (1) ～～Zachary M. Soler MD, MSc ${ }^{4}$ \\ ${ }^{1}$ Division of Rhinology and Sinus Surgery, Department of Otolaryngology-Head and Neck Surgery, University of Virginia, Charlottesville, Virginia, \\ USA \\ ${ }^{2}$ Department of Psychology, Portland State University, Portland, Oregon, USA \\ ${ }^{3}$ Department of Otolaryngology-Head and Neck Surgery, Oregon Health and Science University, Portland, Oregon, USA \\ ${ }^{4}$ Division of Rhinology and Sinus Surgery, Department of Otolaryngology-Head and Neck Surgery, Medical University of South Carolina, Charleston, \\ South Carolina, USA \\ ${ }^{5}$ Department of Surgery, Ralph H. Johnson Veterans Affairs (VA) Medical Center, Charleston, South Carolina, USA \\ ${ }^{6}$ Department of Otolaryngology-Head and Neck Surgery, University of Colorado, Denver, Colorado, USA \\ ${ }^{7}$ Division of Otolaryngology-Head and Neck Surgery, Department of Surgery, University of Utah, Salt Lake City, Utah, USA
}

\section{Correspondence}

Jose L. Mattos, MD, MPH, Division of Rhinology and Sinus Surgery, Department of Otolaryngology-Head and Neck Surgery, University of Virginia, PO Box 800713, Charlottesville, VA 22908, USA.

Email:jm6cb@virginia.edu

\section{Funding information}

Zachary M. Soler, Rodney J. Schlosser, Jess C. Mace, Jeremiah A. Alt, Vijay R. Ramakrishnan, and Timothy L. Smith are supported by grants from the National Institute on Deafness and Other Communication Disorders (NIDCD), one of the National Institutes of Health, Bethesda, MD (R03 DC013651-01; PI: Zachary M. Soler, R01 DC005805; PIs: Timothy L. Smith and Zachary M. Soler, K23DC014747; PI: Vijay R. Ramakrishnan). This funding organization did not contribute to the design or conduct of this study; preparation, review, approval, or decision to submit this manuscript for publication.

\begin{abstract}
Background: The Questionnaire of Olfactory Disorders-Negative Statements (QOD-NS) is a 17-item instrument measuring olfactory-specific quality of life (QOL). However, in clinical research patients can be overwhelmed with multiple questionnaires. We recently developed the 7-item brief QOD-NS (B-QOD). Our objective was to evaluate the psychometric properties of the B-QOD in both the development (D) sample, and in a separate replication (R) sample.

Methods: Testing on $\mathrm{D}(n=203)$ and $\mathrm{R}(n=281)$ samples included initial exploratory factor analysis (EFA), followed by internal reliability, information loss, and confirmatory factor analysis (CFA). Finally, incremental predictive utility analysis (IPUA) was performed by correlating the B-QOD with the 22-item Sino-Nasal Outcome Test (SNOT-22) survey.

Results: EFAs of both D and R demonstrated an underlying single-factor structure (eigenvalue $=4.17$ and 3.57, respectively) with comparable loading factors ( $R>0.30$ for both). B-QOD also had good internal reliability in both $\mathrm{D}$ and $\mathrm{R}$ (Cronbach's alpha $=0.88$ and 0.83 , respectively). Also, there is minimal information loss with B-QOD compared to QOD-NS in both D and $\mathrm{R}(R=0.98$ and 0.96,
\end{abstract}


respectively). CFA indicates that the B-QOD single-factor model has good overall fit as measured by the Comparative Fit Index (CFI) and the Standardized Root Mean Squared Residuals (SRMSR) in the D and R samples (CFI = 0.99 and 0.97; SRMSR $=0.035$ and 0.053). IPUA shows that the QOD-NS offers no additional predictive benefit of SNOT-22 scores when compared with B-QOD.

Conclusion: The 7-item B-QOD captures a structurally coherent and reliable single dimension, with minimal information loss and excellent external predictive utility when compared to the QOD-NS.

\section{KEYWORDS}

olfaction disorders, patient reported outcome measures, quality of life, sinusitis, smell

\section{INTRODUCTION}

Chronic rhinosinusitis (CRS) is a common inflammatory disorder affecting $\sim 5 \%$ to $16 \%$ of North American populations. ${ }^{1}$ Symptoms of CRS are nasal congestion, discolored nasal drainage, facial pain/pressure, and olfactory dysfunction (OD), with OD estimated to affect between $40 \%$ and $80 \%$ of CRS patients in certain study populations. ${ }^{2}$ OD ranks as the second most important symptom for CRS patients after nasal congestion, ${ }^{3}$ and consequently has garnered significant attention in quality of life (QOL) and outcomes research. This has led to the development of instruments that specifically focus on olfactory-specific QOL. ${ }^{4-6}$ In particular, the questionnaire of olfactory disorders negative statements (QOD-NS) is a 17-item validated instrument in the measurement of olfactory-specific QOL, and also demonstrates robust psychometric properties. ${ }^{7,8}$

However, in the clinical and outcomes research setting, patients are frequently inundated with a variety of questionnaires and instruments to complete, and the time required to complete these instruments can be significant. Furthermore, shorter patient-reported outcome metrics (PROMs) have been shown to lead to increased efficiency, reduced patient burden, and greater data quality. ${ }^{9}$ For these reasons, we have previously developed a brief version of the QOD-NS (B-QOD). ${ }^{10}$ The B-QOD was created to intentionally represent all of the subdomains of the QOD-NS, and the B-QOD demonstrates excellent correlation with the total and subdomain scores of the QOD-NS and consists of only seven items which are a subset of the QOD-NS. ${ }^{10}$ Our present objective was to evaluate the psychometric properties of the B-QOD in the CRS population using the initial development sample and also in a separate replication sample. In the remainder, we refer to these samples as the development sample (D) and the replication sample (R), respectively.

\section{SUBJECTS AND METHODS}

\section{Sample populations}

The purpose of these analyses was to evaluate the psychometric properties of the B-QOD using the initial D sample (enrollment years: 2011-2016; $n=203$ ) and in the R sample (enrollment years: 2016-2019; $n=281$ ). Study enrollment originated from two observational, prospective research investigations of human subjects funded by the National Institute on Deafness and Other Communication Disorders (NIDCD, Bethesda, MD, USA). Study participants were recruited at varying times from heterogeneous patient populations presenting to academic, rhinology centers located in North America: Oregon Health \& Science University (Portland, OR, USA), the Medical University of South Carolina (MUSC, Charleston, SC, USA), Stanford University (Palo Alto, CA, USA), the University of Utah (Salt Lake City, UT, USA), the University of Colorado (Aurora, CO, USA), the University of Calgary (Calgary, Alberta, Canada), and the University of Virginia (Charlottesville, VA, USA). The Institutional Review Board at each performance site provide ethical review and oversight of this minimal risk study. Table 1 provides the demographic and disease-specific characteristics of these cohorts.

Symptomatic, adult study participants received a confirmed diagnosis of symptomatic and medically recalcitrant CRS, with nasal polyposis (CRSwNP) or without nasal polyposis (CRSsNP), from a fellowship-trained rhinologist following criteria established by current practice guidelines. ${ }^{11}$ Participants presented with symptoms including, but not limited to, nasal congestion, mucopurulent drainage, facial pain/pressure, and olfactory impairment. All patients provided written, informed consent to ensure voluntary participation for this minimal risk study. 
TA B L E 1 Demographic and disease-specific characteristics of the D and R samples

\begin{tabular}{|c|c|c|c|c|}
\hline Characteristics & $\begin{array}{c}\text { Sample D } \\
(n=203)\end{array}$ & $\begin{array}{c}\text { Sample R } \\
(n=281)\end{array}$ & Test statistic & $p$ \\
\hline Age (years), mean $\pm S D$ & $50.5 \pm 15.8$ & $49.5 \pm 16.6$ & $t=0.62$ & 0.538 \\
\hline Female, $n(\%)$ & $102(51)$ & $144(51)$ & & \\
\hline White/Caucasian, $n(\%)$ & $168(84)$ & $249(89)$ & $\chi^{2}=3.39$ & 0.066 \\
\hline Hispanic/Latino, $n(\%)$ & $11(6)$ & $17(6)$ & $\chi^{2}=0.04$ & 0.845 \\
\hline Prior ESS, $n(\%)$ & $120(62)$ & $124(44)$ & $\chi^{2}=13.70$ & $<0.001$ \\
\hline Nasal polyposis, $n(\%)$ & $76(39)$ & $148(53)$ & $\chi^{2}=8.89$ & 0.003 \\
\hline Asthma, $n(\%)$ & $88(45)$ & $125(45)$ & $\chi^{2}=0.01$ & 0.917 \\
\hline SIT total score, mean \pm SD & $27.6 \pm 9.6$ & - & - & - \\
\hline Sniffin' Sticks TDI score, mean \pm SD & - & $23.0 \pm 9.4$ & - & - \\
\hline QOD-NS total score, mean \pm SD & $14.4 \pm 12.9$ & $13.4 \pm 10.7$ & $t=0.93$ & 0.478 \\
\hline SNOT-22 total score, mean \pm SD & $53.4 \pm 21.7$ & $46.1 \pm 21.1$ & $t=3.70$ & $<0.001$ \\
\hline
\end{tabular}

Abbreviations: BSIT, Brief Smell Identification Test; CT, computed tomography; D, development; ESS, endoscopic sinus surgery; QOD-NS, Questionnaire of Olfactory Disorders-Negative Statements; R, replication; SD, standard deviation; SIT, Smell Identification Test; SNOT-22, 22-item Sino-Nasal Outcome Test; TDI score, threshold discrimination and identification composite score.

\section{Exclusion criteria}

Study participants were excluded from final cohort selection if they failed to complete QOD-NS sample survey evaluations at the time of study enrollment. Additionally, any study participants with comorbid primary ciliary dyskinesia or cystic fibrosis (CF) were excluded due to possible disparity in disease presentation and/or treatment approach.

\section{Analytic strategy and biostatistics}

First, we performed exploratory factor analyses using the B-QOD data from the two samples in order to examine the number of underlying constructs that the B-QOD might be representing, with the hypothesis that the BQOD represents a single factor. We present standardized factor loadings, which estimate the correlation between the underlying factor and the items; residual variances estimate the variance in item scores not attributable to the underlying factor. An item is deemed a "salient" indicator if the standardized factor loading for that item exceeds $|0.32|$ because the factor "explains" $>10 \%$ of the variance in the item; items with loadings greater than $0.71,0.63$, and 0.55 are considered "excellent," "very good," and "good," respectively. ${ }^{12}$ We then checked the internal consistency and reliability of the estimates achieved by the B-QOD. To do this, we used Cronbach's alpha. Nunnally and Bernstein ${ }^{13}$ provide guidelines for judging the adequacy of reliability estimates stating that estimates exceeding 0.70 are considered adequate for use in research and estimates exceeding 0.80 are considered good. Interested readers desiring to learn more about psychometric analysis and the statistics involved could consult Nunnally and Bernstein's ${ }^{13}$ and McDonald's ${ }^{14}$ books on the topic.

Next we explored the degree of possible information loss between the B-QOD and the QOD-NS. Because the B-QOD is based on seven of the 17 items in the QOD-NS it is possible that in using fewer than half of the original items, the B-QOD may omit important information from overall scale scores using all 17 items. To evaluate the degree of information loss, we correlated scores between the full 17-item measure with the 7-item B-QOD.

After these steps, a confirmatory factor analysis (CFA) model is needed to evaluate the single-factor hypothesis directly. For this end, we used the CFA model for ordinal indicators in Mplus software. ${ }^{15}$ We then utilized the comparative fit index (CFI), which indicates good fit when values exceed $0.95 ;{ }^{16}$ the root mean square error of approximation (RMSEA) indicates good fit with values $<0.05$ and poor fit with values $>0.10^{16}$; the standardized root mean square residual (SRMR) indicates good fit when 
TA B L E 2 Standardized factor loadings for B-QOD items in the D sample and R sample from exploratory factor analysis models

$\begin{array}{lcc}\text { Item } & \text { Sample D } & \text { Sample R } \\ \text { QOD-NS 1 “Restaurants” } & 0.74 & 0.70 \\ \text { QOD-NS 13 “Worried” } & 0.65 & 0.60 \\ \text { QOD-NS 27 “Relax” } & 0.69 & 0.69 \\ \text { QOD-NS 33 “Isolated” } & 0.77 & 0.67 \\ \text { QOD-NS 37 “Eat less” } & 0.69 & 0.50 \\ \text { QOD-NS 42 “Daily activities” } & 0.79 & 0.73 \\ \text { QOD-NS 49 “Angry” } & 0.76 & 0.67\end{array}$

Abbreviations: B-QOD, Brief Questionnaire of Olfactory Disorders; D, development; QOD-NS, Questionnaire of Olfactory Disorders-Negative Statements; $\mathrm{R}$, replication.

values are $<0.05$. We also calculated standardized factor loadings from the confirmatory analysis on both samples.

Finally, we performed an analysis of incremental predictive utility of the QOD-NS compared to the B-QOD scores for a common QOL measure in the CRS literature, the 22-item Sino-Nasal Outcome Test (SNOT-22). ${ }^{17}$ First, we descriptively computed and compared the correlations between QOD-NS and B-QOD scores with SNOT-22 total scale scores in the D and R to test whether QOD-NS scores have additional incremental predictive utility in the prediction of SNOT-22 total scores, a hierarchical linear regression approach was used. In the first step, SNOT-22 total scores were regressed on B-QOD scores; in the second step, QOD-NS scores were added as a predictor to the model. The statistic of interest is the change in $R^{2}$ from step 1 to step 2.

\section{RESULTS}

\section{Exploratory factor analyses}

The two exploratory factor analyses (EFAs) yielded similar results about the underlying structure of the B-QOD. Globally, both EFAs exhibited a single, dominant dimension of variability for the underlying factor structure with only a single eigenvalue greater than one (sample D 1st eigenvalue $=4.17$; sample $\mathrm{R} 1$ st eigenvalue $=3.57$ ). Table 2 provides the standardized factor loadings from the EFAs for the two samples. The standardized factor loadings are substantively similar in size across the two samples. As would be expected, the standardized factor loadings tend to be a larger in D, which illustrates the importance of evaluating psychometric properties on an independent sample over and above the development sample. Despite this slight attenuation, the standardized factor loadings in sample $\mathrm{R}$ are all sizable and far exceed the threshold for a salient loading (i.e., a loading of 0.30 or greater).
TA B L E 3 Cronbach's alpha internal consistency reliability estimates for the B-QOD in the D sample and R sample

$\begin{array}{lcc}\text { Item } & \text { Sample D } & \text { Sample R } \\ \text { Cronbach's alpha } & 0.88 & 0.83\end{array}$

Abbreviations: B-QOD, Brief Questionnaire of Olfactory Disorders; D, development; R, replication.

TA B L E 4 Correlation between the QOD-NS scores and B-QOD scores in the D sample and R sample

\begin{tabular}{lcc} 
Item & Sample D & Sample R \\
Correlation & $0.98^{*}$ & $0.96^{*}$ \\
\hline
\end{tabular}

Abbreviations: B-QOD, Brief Questionnaire of Olfactory Disorders; D, development; QOD-NS, Questionnaire of Olfactory Disorders-Negative Statements; $\mathrm{R}$, replication. ${ }^{*} p<0.05$

\section{Internal consistency reliability estimates}

Given that the EFAs strongly support the single-factor structure of the B-QOD, we next turn to how reliably these items measure that single latent dimension. Table 3 provides the Cronbach's alpha estimates for the two samples. The reliability estimates for the B-QOD far exceed the criterion value considered good in both samples.

\section{Information loss between the QOD-NS and B-QOD}

Table 4 provides these correlations for the two samples between QOD-NS and B-QOD scores. These correlations should be considered very large and suggest that little information is lost when using the B-QOD rather than all 17 of the QOD-NS items.

\section{CFA of the B-QOD items}

The fit statistics for the models, along with the CFI, SRMR, and RMSEA statistics in the two samples appear in Table 5.

TA B L E 5 Fit statistics for confirmatory factor analysis model with categorical indicators for the B-QOD in the D and R samples

$\begin{array}{lcc}\text { Item } & \text { Sample D } & \text { Sample R } \\ \text { Chi-square statistic (DF) } & 21.14(11) & 39.54(12) \\ \text { CFI } & 0.991 & 0.967 \\ \text { RMSEA } & 0.067 & 0.090 \\ \text { SRMR } & 0.035 & 0.053\end{array}$

Abbreviations: B-QOD, Brief Questionnaire of Olfactory Disorders; CFI, comparative fit index; D, development; DF, model degrees of freedom; R, replication; RMSEA, root mean square error of approximation; SRMR, standardized root mean square residual. 
TA B L E 6 Standardized factor loadings for B-QOD items in the D sample and R sample from single-factor confirmatory factor analysis models with categorical indicators

\begin{tabular}{|c|c|c|c|c|}
\hline \multirow[b]{2}{*}{ Item } & \multicolumn{2}{|c|}{ Sample D } & \multicolumn{2}{|c|}{ Sample R } \\
\hline & Loading (SE) & Residual variance & Loading (SE) & Residual variance \\
\hline QOD-NS 1 “Restaurants” & $0.842(0.036)$ & 0.291 & $0.804(.038)$ & 0.353 \\
\hline QOD-NS 13 “Worried” & $0.767(0.045)$ & 0.412 & $0.703(.046)$ & 0.505 \\
\hline QOD-NS 27 “Relax” & $0.804(0.041)$ & 0.353 & $0.805(.040)$ & 0.352 \\
\hline QOD-NS 33 “Isolated” & $0.859(0.033)$ & 0.262 & $0.778(.040)$ & 0.395 \\
\hline QOD-NS 49 “Angry” & $0.851(0.035)$ & 0.276 & $0.771(.040)$ & 0.405 \\
\hline
\end{tabular}

Abbreviations: B-QOD, Brief Questionnaire of Olfactory Disorders; D, development; QOD-NS, Questionnaire of Olfactory Disorders-Negative Statements; R, replication; SE, standard error.

TA B L E 7 Correlation between the QOD-NS scores and B-QOD scores and SNOT-22 total scores in the D sample and R sample

\begin{tabular}{lcc} 
Item & $\begin{array}{c}\text { Sample D } \\
\text { SNOT-22 total } \\
\text { scores }\end{array}$ & $\begin{array}{c}\text { Sample R } \\
\text { SNOT-22 total } \\
\text { scores }\end{array}$ \\
QOD-NS scores & $0.42^{*}$ & $0.37^{*}$ \\
B-QOD scores & $0.40^{*}$ & $0.38^{*}$ \\
\hline
\end{tabular}

Abbreviations: B-QOD, Brief Questionnaire of Olfactory Disorders; D, development; QOD-NS, Questionnaire of Olfactory Disorders-Negative Statements; $\mathrm{R}$, replication; SNOT-22, 22-item Sino-Nasal Outcome Test. ${ }^{*} p<0.05$.

Overall, these results suggest that the single-factor hypothesis for B-QOD is a useful approximation to the data. Given the adequate level of fit of the model, we now evaluated the standardized factor loadings from the CFA models in both samples. These are presented in Table 6. These loadings are all large in size indicating that each item strongly relates to the underlying single factor.

\section{Analysis of incremental predictive utility of QOD-NS over B-QOD}

Table 7 displays the correlations between QOD-NS and BQOD scores with SNOT-22 scores in both samples; we note that these correlations are similar in magnitude. These correlations provide evidence of the criterion-related validity of the QOD-NS and the B-QOD. For the assessment of incremental predictive utility, QOD-NS scores did not significantly increase the variance explained in SNOT22 scores $[\Delta \mathrm{R} 2=0.014, \mathrm{~F}(1,200)=3.376, p=0.068]$ in D when B-QOD is controlled for statistically; similarly, in R, QOD-NS scores did not significantly increase the variance explained in SNOT-22 scores $[\Delta \mathrm{R} 2<0.001$, $\mathrm{F}(1,277)=0.046, p=0.830]$ when $\mathrm{B}-\mathrm{QOD}$ is controlled for statistically. Thus, we conclude that QOD-NS scores offer no statistically significant benefit in the prediction of
SNOT-22 total scores over and above the information available from the B-QOD.

\section{DISCUSSION}

We have previously reported on our development of the $\mathrm{B}-\mathrm{QOD},{ }^{10}$ which is a subset of seven items from the original QOD-NS. Our initial report confirmed that the B-QOD purposefully included all of the subdomains of the original QOD-NS, and B-QOD scores have good correlation with total QOD-NS scores as well as the subdomains scores that are captured by the QOD-NS. ${ }^{7,10}$ Furthermore, the QODNS has been previously validated in CRS patients, and this validation included CRS patients along the entire spectrum of olfactory ability. ${ }^{18}$ As such, we maintain a similar approach to the creation and validation of the B-QOD in CRS patients ranging from normosmics to anosmics. However, given the substantial reduction in the number of items from the QOD-NS to the B-QOD, it is possible that the shortened instrument may not have all of the desired psychometric properties. Psychometric properties refer to the validity and reliability of a measurement tool. ${ }^{13} \mathrm{~A}$ valid tool is one that measures what is says it is going to measure, whereas a reliable tool consistently measures the same construct.

Our analysis has shown that the B-QOD is both valid and reliable. The B-QOD has good internal validity, as shown by the exploratory and confirmatory factor analyses demonstrating a single unified construct (Tables 2, 5, and 6). Furthermore, our results show that there is no loss of information when comparing the B-QOD to the original QOD-NS (Table 4). The external validity of the B-QOD is confirmed by our analysis demonstrating good correlations with SNOT-22 scores, and the fact that the original QOD-NS does not add any predictive utility to SNOT-22 scores when compared to the B-QOD (Table 7). Furthermore, the Cronbach's alpha analysis confirms the internal 
consistency and reliability of the B-QOD (Table 3). It is important to note that the results hold true both in the original cohort used to develop the B-QOD, but also notably in an entirely separate replication cohort. The consistent findings between the performance of the B-QOD in two independent cohorts add further evidence that this instrument is valid and reliable and should be considered for use.

The B-QOD is likely most useful for situations where there is little time for patient to completely questionnaire (i.e., a busy clinical setting) or where patients may be overburdened by numerous surveys or questionnaires (i.e., prospective clinical research settings). In reality, the majority of patients or research subjects are likely encompassed by these two scenarios, and so we expected that the BQOD could be useful for a wide variety of future projects or investigations. However, some investigators may desire the greater granularity that the original QOD-NS allows, particularly the ability to study the different subdomains or factors captured by the QOD-NS. ${ }^{7}$

This study has several limitations. First, the participants in both $\mathrm{D}$ and $\mathrm{R}$ were all CRS patients, and it is possible that the instruments psychometric properties may be different in different populations. Also, the participants had high associated disease severity and were assessed through tertiary practices, and therefore these results may not be externally generalizable to all CRS patients. Further validation and study of our shortened version in other populations with different etiologies for olfactory dysfunction (i.e., again, postinfectious, posttraumatic, etc.) of the will be necessary to further confirm its clinical utility and validity.

\section{CONCLUSION}

The results of this psychometric investigation indicate that data from B-QOD capture a structurally coherent single latent dimension, that the $\mathrm{B}-\mathrm{QOD}$ reliably measures this dimension, that little information is lost when using the seven items of the B-QOD relative to using all 17 items of the QOD-NS, and that the B-QOD has excellent external predictive utility when compared to the QOD-NS. In settings where olfactory-specific QOL is of interest but survey burden is a concern, or where rapid completion of PROMs is of interest, the B-QOD should be considered for use due to its few items and excellent psychometric properties.

\section{CONFLICTS OF INTEREST}

Zachary M. Soler: Olympus, OptiNose, Regeneron, Healthy Humming, and Novartis, consultant (not affiliated with this study). Jeremiah A. Alt: Medtronic and
GlycoMira Therapeutics, consultant (not affiliated with this study). Rodney J. Schlosser: OptiNose, Olympus, Stryker, Regeneron, and Healthy Humming, consultant (not affiliated with this study). Vijay R. Ramakrishnan: OptiNose and Medtronic, Inc, consultant (not affiliated with this study). Daniel M. Beswick: Medtronic, consultant (not affiliated with this study).

\section{O R C I D}

Jose L. Mattos MD, MPH (1) https://orcid.org/0000-00018766-1626

Daniel M. Beswick MD (1D https://orcid.org/0000-00018612-5442

Spencer C. Payne MD (1) https://orcid.org/0000-0001-97364561

Timothy L. Smith MD, MPH (1) https://orcid.org/0000-

0002-6424-7083

\section{REFERENCES}

1. Smith KA, Orlandi RR, Rudmik L. Cost of adult chronic rhinosinusitis: a systematic review. Laryngoscope. 2015;125(7):1547-1556.

2. Soler ZM, Smith TL, Alt JA, Ramakrishnan VR, Mace JC, Schlosser RJ. Olfactory-specific quality of life outcomes after endoscopic sinus surgery. Int Forum Allergy Rhinol. 2016;6(4):407-413.

3. Mattos JL, Rudmik L, Schlosser RJ, et al. Symptom importance, patient expectations, and satisfaction in chronic rhinosinusitis. Int Forum Allergy Rhinol. 2019;9(6):593-600.

4. Soler ZM, Kohli P, Storck KA, Schlosser RJ. Olfactory impairment in chronic rhinosinusitis using threshold, discrimination, and identification scores. Chem. Senses. 2016;41(9):713-719.

5. DeConde AS, Mace JC, Alt JA, Schlosser RJ, Smith TL, Soler ZM. Comparative effectiveness of medical and surgical therapy on olfaction in chronic rhinosinusitis: a prospective, multiinstitutional study. Int Forum Allergy Rhinol. 2014;4(9):725-733.

6. Patel ZM, Thamboo A, Rudmik L, Nayak JV, Smith TL, Hwang $\mathrm{PH}$. Surgical therapy vs continued medical therapy for medically refractory chronic rhinosinusitis: a systematic review and metaanalysis. Int Forum Allergy Rhinol. 2017;7(2):119-127.

7. Mattos JL, Schlosser RJ, DeConde AS, et al. Factor analysis of the questionnaire of olfactory disorders in patients with chronic rhinosinusitis. Int Forum Allergy Rhinol. 2018;8(7):777-782.

8. Rudmik L, Hopkins C, Peters A, Smith TL, Schlosser RJ, Soler ZM. Patient-reported outcome measures for adult chronic rhinosinusitis: a systematic review and quality assessment. J Allergy Clin Immunol. 2015;136(6):1532-1540.e2.

9. Harel D, Baron M. Methods for shortening patient-reported outcome measures. Statis Methods Med Res. 2018;28(10-11):29923011.

10. Mattos JL, Edwards C, Schlosser RJ, et al. A brief version of the questionnaire of olfactory disorders in patients with chronic rhinosinusitis. Int Forum Allergy Rhinol. 2019;9(10):1144-1150.

11. Rosenfeld RM, Piccirillo JF, Chandrasekhar SS, et al. Clinical practice guideline (Update): adult sinusitis executive summary. Otolaryngol Head Neck Surg. 2015;152(4):598-609.

12. Comrey AL, Lee HB. A First Course in Factor Analysis. 2nd ed. Mahwah, NJ: Erlbaum; 1992. 
13. Nunnally J, Bernstein I. Psychometric Theory. 3rd ed. New York: McGraw-Hill; 1994.

14. McDonald RP. Test Theory: a Unified Treatment. Mahwah, NJ: Erlbaum; 1999.

15. Muthén LK, Muthén BO. Mplus. Statistical Analysis With Latent Variables. User's Guide. Los Angeles, CA: Muthén \& Muthén; 2017. https://www.statmodel.com/download/usersguide/Mplus UserGuideVer_8.pdf. Accessed April 11, 2021.

16. $\mathrm{Hu} \mathrm{L}$, Bentler PM. Cutoff criteria for fit indexes in covariance structure analysis: conventional criteria versus new alternatives. Struct Equ Modeling. 1999;6(1):1-55.

17. Hopkins C, Gillett S, Slack R, Lund VJ, Browne JP. Psychometric validity of the 22-item sinonasal outcome test. Clin Otolaryngol. 2009;34(5):447-454.
18. Simopoulos E, Katotomichelakis M, Gouveris H, Tripsianis G, Livaditis M, Danielides V. Olfaction-associated quality of life in chronic rhinosinusitis: adaptation and validation of an olfactionspecific questionnaire. Laryngoscope. 2012;122(7):1450-1454.

How to cite this article: Mattos JL, Bodner TE, Mace JC, et al. Psychometric properties of the brief version of the questionnaire of olfactory disorders in patients with chronic rhinosinusitis. Int Forum Allergy Rhinol. 2021;1-7.

https://doi.org/10.1002/alr.22800 